\title{
Study the Effect of Barium Sulphate on Optical Properties of Polyvinyl Alcohol (PVA)
}

\author{
Angham.G.Hadi ${ }^{1}$, Farhan Lafta ${ }^{2}$, Ahmed Hashim ${ }^{1, *}$, Hussein Hakim ${ }^{1}$, Abbas I. O. Al-Zuheiry ${ }^{1}$, \\ Saba R. Salman ${ }^{1}$, Hind Ahmed ${ }^{1}$ \\ ${ }^{1}$ Babylon university, Iraq \\ ${ }^{2}$ Ministry of Science and Technology,Iraq \\ *Corresponding Author: ahmed_taay@yahoo.com
}

Copyright (C) 2013 Horizon Research Publishing All rights reserved.

\begin{abstract}
The present work is concerned to study the optical constants of the $\mathrm{PVA} / \mathrm{BaSO}_{4}$ composites at different concentrations of barium sulphate. The samples are prepared by casting method technique with different proportion of PVA and $\mathrm{BaSO}_{4}$. The absorption and transmission spectra have been recorded at the wavelength ranges $(200-800) \mathrm{nm}$ by using (UV/160/Shimadzu spectrophotometer). The results show that (The absorption coefficient, extinction coefficient and energy gap of the indirect allowed and forbidden transition) vary with the concentration of barium sulphate $\mathrm{BaSO}_{4}$ dopant.
\end{abstract}

Keywords Polymer, PVA, Barium Sulfate, Optical Properties, Absorbance

\section{Introduction}

In the recent years, studies on the electrical and optical properties of polymers have attracted much attention in view of their application in electronic and optical devices. Electrical conduction in polymers has been studied aiming to understand the nature of the charge transport prevalent in these materials while the optical properties are aimed at achieving better reflection, antireflection, interference and polarization properties [1,2].

One of the important classes of polymer electrolytes is polar polymer \{like Polyethylene oxide (PEO), Polypropylene oxide (PPO), polyvinyl alcohol (PVA), etc $\}$. In this paper, we have used PVA as a host polymer because PVA is semi-crystalline polymer and has very important applications due to the role of $\mathrm{OH}$ group and hydrogen bonds [3]. The stability of polymer thin films on solid substrates is of great technological importance in applications ranging from protective coatings to paintings, semiconductors, and micro- and optoelectronic devices $[4,5,6]$.

Optical polymers have attracted considerable attention in recent years because of their important industrial applications such: solar cells, diodes, filters of light, ... . etc. The present study will help in understanding the effect of different concentrations of barium salt on the optical parameters.

\section{Experimental Part}

The polymer(PVA) was dissolved in distill water by using magnetic stirrer in mixing process to get homogeneous solution at $90^{\circ} \mathrm{C}$, then the solution was cooled at room temperature. The barium sulphate was add to the polymer with different concentration are (1,2 and 3) wt.\% and mixed for 10 minutes to get more homogenous solution, then the solution was poured in petri dish $(5.5 \mathrm{~cm})$. placed on plate form and let it to dry at room temperature. The films were ready which cut into pieces for characterization by measuring The absorption and transmission by using UV/160/Shimadzu spectrophotometer.

\section{Results and Discussion}

\subsection{The Absorbance of Composites}

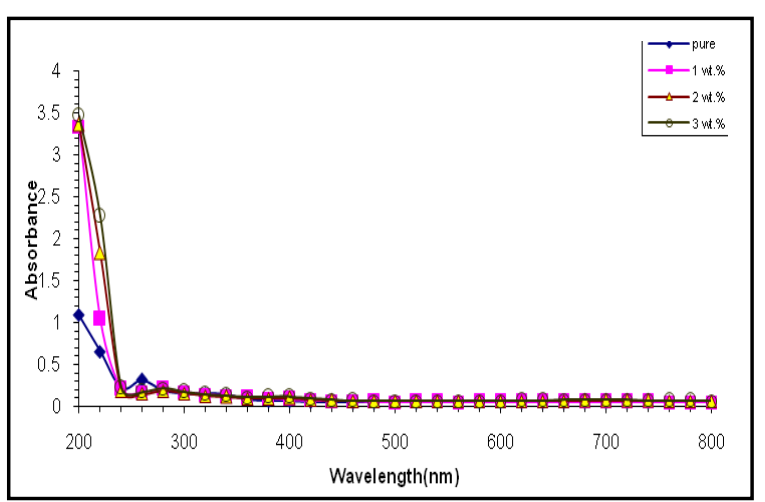

Figure 1. The variation of optical absorbance for(PVA-BaSO ${ }_{4}$ )composite with wavelength 
Fig.1 shows the relationship between absorbance of

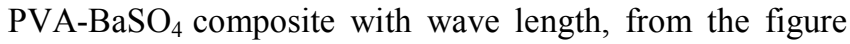
shows that the absorption increases as a result of filler addition but no shift in the peak position, which means the addition to pure polymer does not change the chemical structure of the material but new physical mixture is formed the absorbance stay constant after $240 \mathrm{~nm}$.

\subsection{The Absorption Coefficient and Energy Gap of Composites}

Fig. 2 shows the optical absorption spectrum of composite for different impurities quantities, it was found that the composite have a low absorption coefficient at a small photon energy then increase at different rates dependence on the composite structure. The pure sample had low absorption coefficient this may be as a result of low crystalinity. Analysis of optical absorption spectra could reveal the energy gap Eg between the conduction band (CB) and the valence band (VB) due to direct and indirect transitions of both crystalline and amorphous materials.

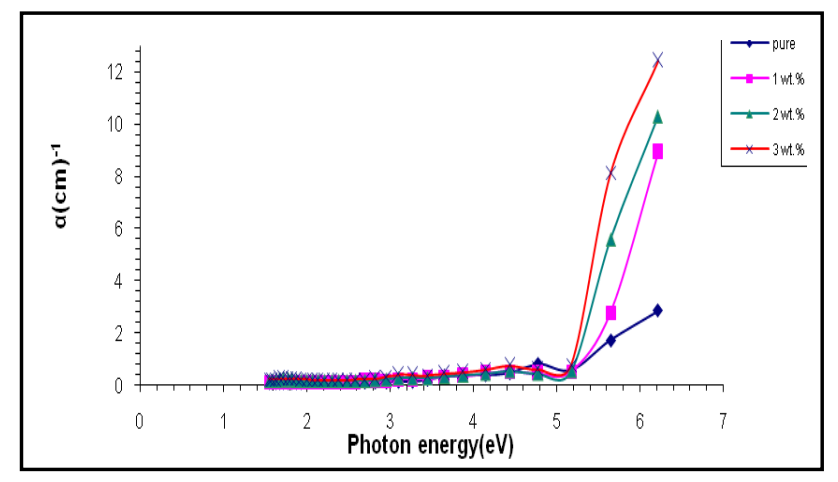

Figure 2. The absorption coefficient for(PVA-BaSO 4 ) composite with various photon energy

The absorption coefficient $(\alpha)$ was calculated in the fundamental absorption region from the following equation[6]:

$$
\alpha=2.303 \frac{A}{d}
$$

Where : A is absorbance and (d) is the thickness of sample.

Fig .3 and Fig. 4 represented the indirect transition, the energy gab values dependence in general on the crystal structure of the composites and on the arrangement and distribution way of atoms in the crystal lattice. The optical energy gap (Eg) of the thin films has been determined from absorption coefficient data as a function of photon energy. According to the generally accepted "non-direct transition model" for amorphous semiconductors proposed by Tauc [7].

$$
\alpha h v=B\left(h v-E_{g}\right)^{r}
$$

Where $\mathrm{B}$ is a constant related to the properties of the valance band and conduction band, hv is the photon energy, $\mathrm{E}_{\mathrm{g}}$ is the optical energy band gap, $r=2$,or3 for indirect allowed and indirect forbidden transition .

From the linear plots of $(\alpha h v)^{l / r}$ against (h $\left.v\right)$ for these samples as shown in figure3, the optical energy gap has been determined from the intercepts of extrapolations to zero with the photon energy axis $(\alpha h v)^{1 / \mathrm{r}} \rightarrow 0$ From the results obtained it is seen that an decrease of concentration of $\mathrm{BaSO}_{4}$ in the system leads to an increase in the optical band gap. A decrease in the energy band gap with increasing $\mathrm{BaSO}_{4}$ concentration may be attributed to an increase in structural disorder of the polymer films with increasing $\mathrm{BaSO}_{4}$ concentration.

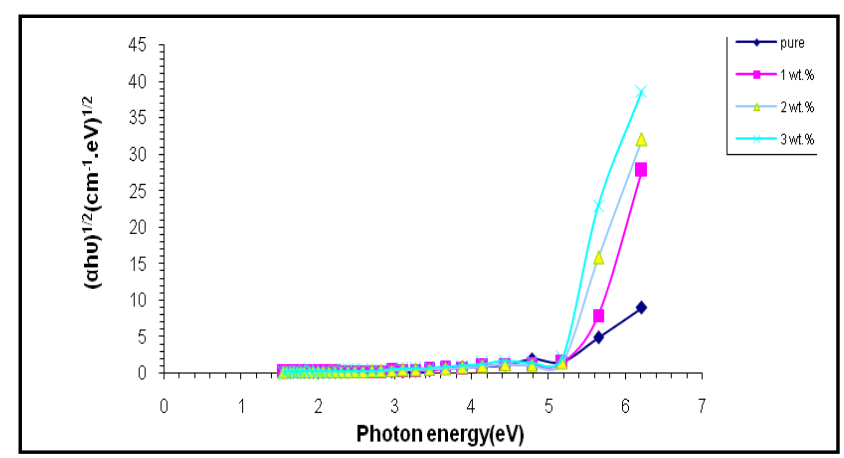

Figure 3. The relationship between $(\mathrm{ahu})^{1 / 2}(\mathrm{~cm}-1 . \mathrm{eV})^{1 / 2}$ and photon energy of PVA-NiCl2 composites

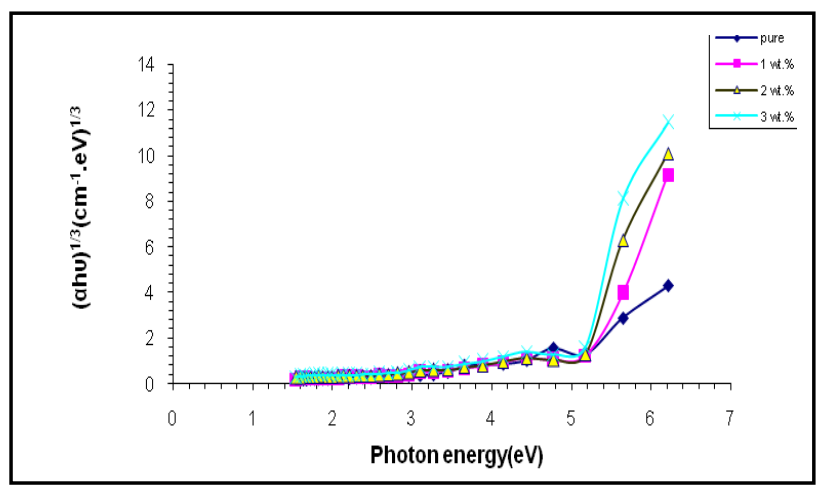

Figure 4. The relationship $(\mathrm{ahu})^{1 / 3}(\mathrm{~cm}-1 . \mathrm{eV})^{1 / 3}$ and photon energy of PVA-BaSO 4 composites

\subsection{Refractive Index and Extinction Coefficient}

The extinction coefficient can be calculated by the relation [5]:

$\mathrm{k}=\alpha \lambda / 4 \pi$

Where $\lambda$ is the wavelength and $\alpha$ is the absorption coefficient.

The refractive index of the films was calculated by the following equation [5]:

$$
\mathrm{n}=\left[4 \mathrm{R} /(\mathrm{R}-1)^{2}-\mathrm{K}^{2}\right]-(\mathrm{R}-1) /(\mathrm{R}-1) \ldots
$$

where $\mathrm{R}$ the reflectance and $\mathrm{k}$ the extinction coefficient and 
define as above.

Fig. 5 shows the variation of extinction coefficient(k) with of the composite with photon energy, the values increase exponentially with increasing photon energy. This increase indicates that the electromagnetic radiation passing through the material is faster in the low photon energy.

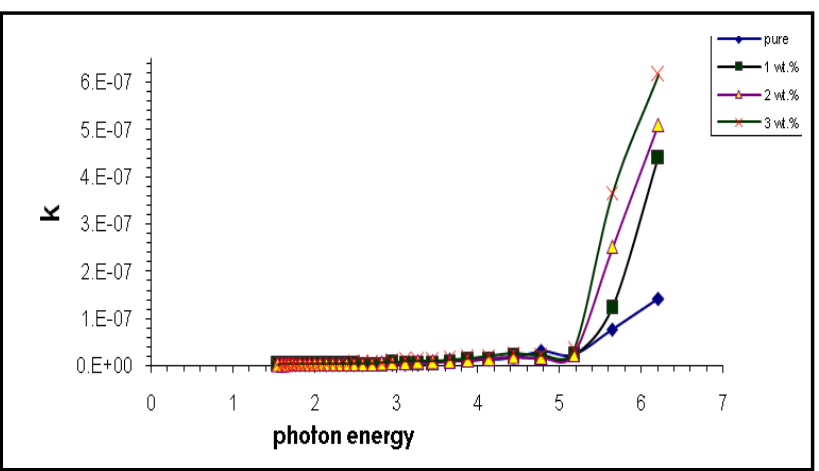

Figure 5. The extinction for( $\left(\mathrm{PVA}-\mathrm{BaSO}_{4}\right)$ composite with various photon energy

Fig.6 represent the variation of the refractive index(n) with the incident photon energy, the variation is simple in the low energy region while it increased in the high photon energy region, this behavior may be as a result to the variation of the absorption coefficient which leads to spectral deviation in the location of the charge polarization at the attenuation coefficient due to the loses in the energy of the electron transition between the energy bands.

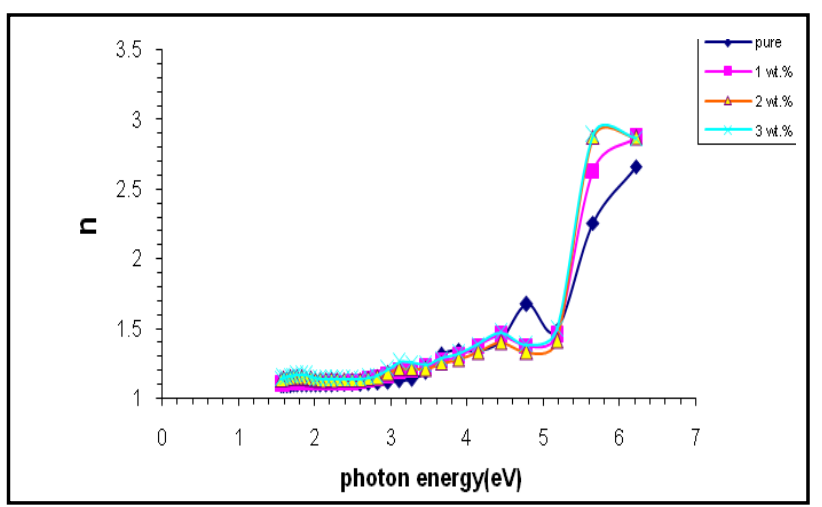

Figure 6. The relationship between refractive index for $\left(\mathrm{PVA}^{-} \mathrm{BaSO}_{4}\right)$ composite with photon energy

\subsection{Dielectric Constants}

Fig. 7 and Fig.8 represented the real and imaginary parts of the dielectric constant, in the real part the variation it was very clear spatially in the high impurities concentration this may be due to the absence the resonance between the frequencies of the incident photon energy (electromagnetic and the induced dipoles in the composite), while in the imaginary part there was an absorption to the energy of the incident photon energy, so the variation nearly constant until it reaches to the high photon energy. The pure composite shows the smaller variation.

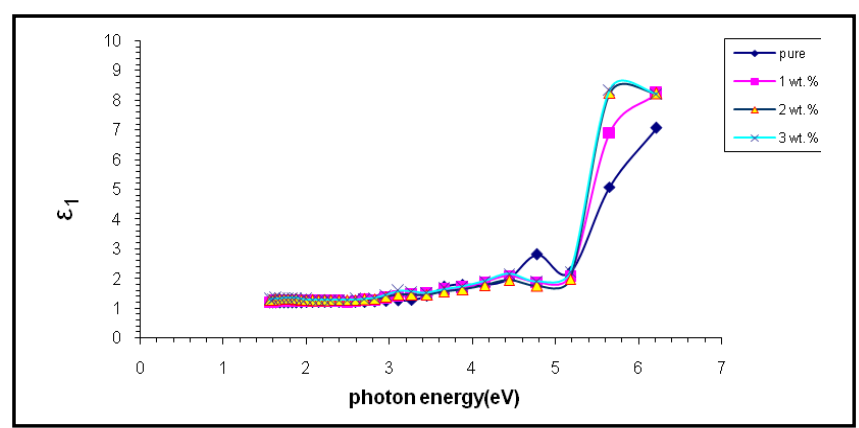

Figure 7. The variation of real part of dielectric constant(PVA-BaSO4)composite with photon energy

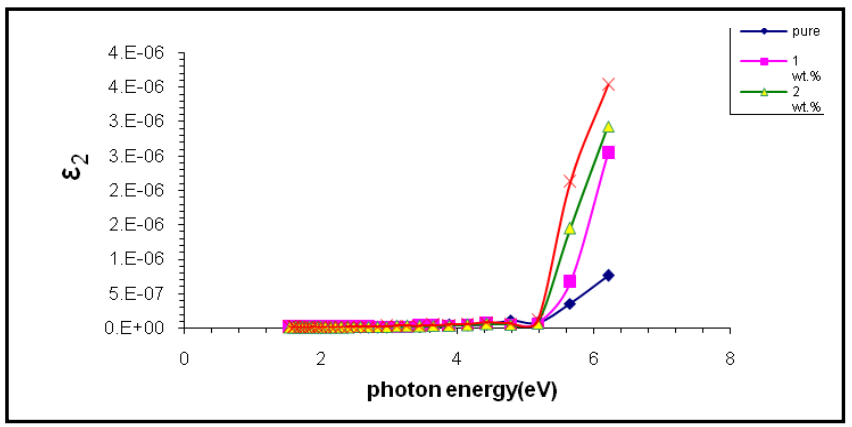

Figure 8. The variation of imaginary part of dielectric

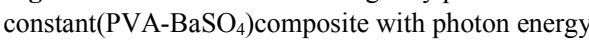

\section{Conclusions}

- The absorbance of composites is very large in the uv, region. The absorption coefficient is smaller and stable in the low photon energy.

- The absorbance of polymer increases with the increase of concentrations of barium sulphate.

- The absorption coefficient and extinction coefficient are increased as a result of the scattering centers in the composites. The values of the refractive index(n) and extinction coefficient of the composites increase exponentially with increasing photon energy

- The real and imaginary dielectric constants show the exponential increase with increasing of the incident photon energy.

- The optical constants of polyvinyl alcohol (absorption coefficient, refractive index extinction coefficient, real and imaginary dielectric constant are increasing with the increase of concentrations of barium sulphate.

- The energy band gap of polyvinyl alcohol decreases with the increase of concentrations of barium sulphate.

\section{REFERENCES}


[1] C. Uma Devi, A.K. Sharma, and V.V.R.N. Rao, 2002 "Electrical and optical properties of pure and silver nitrate-doped polyvinyl alcohol films", Materials Letters, 56, 167-174 .

[2] Lyly n. I, Habibah Z. 2012, Influence of Doping Concentration on Dielectric, Optical, and Morphological Properties', Advances in Materials Science and Engineering, Article ID 605673, 5 pages.

[3] A. Tawansi, A. El-Khodary, and M.M. Abdelnaby, 2005 "A study of the physical properties of $\mathrm{FeCl} 3$ filled PVA", Current
Applied Physics, 5, 572-578 .

[4] Oslanec, R.; Costa, A. C. 2000 ; Composto, R. J. Macromolecules, 33, 5505-5512.

[5] Reiter, G. Macromolecules 1994, 27, 3046-3052.

[6] Adnan KURT, 2010, Influence of $\mathrm{AlCl} 3$ on the optical properties of new film, Turk J Chem, 34, 67-79.

[7] Kathalingam A ., 2007, "Materials Chemistry and physics, vol.106,No. 215 